\title{
Design and Implementation of the MESH Services Platform
}

\author{
Harold J. Batteram \\ Lucent Technologies \\ P.O. Box 18 \\ 1270 AA Huizen NL \\ batteram@lucent.com
}

\author{
John-Luc Bakker \\ Lucent Technologies \\ P.O. Box 18 \\ 1270 AA Huizen NL \\ jlbakker@lucent.com
}

\author{
Jack P.C. Verhoosel \\ Telematics Institute \\ P.O. Box 58 \\ 7500 AN Enschede NL \\ J.Verhoosel@telin.nl
}

\author{
Nikolay K. Diakov \\ CTIT \\ P.O. Box 217 \\ 7500 AE Enschede NL \\ Diakov@ctit.utwente.nl
}

\begin{abstract}
Industry acceptance of TINA (Telecommunications Information Networking Architecture) will depend heavily on both the evaluation of working systems that implement this architecture, and on the experiences obtained during the design and implementation of these systems.

During the $\mathrm{MESH}^{\varphi}$ (Multimedia services on the Electronic Super Highway) project, a TINA based platform for networked multimedia services has been developed and evaluated. This platform, referred to as the MESH platform, implements major parts of the TINA Service Architecture version 5.0 and the TINA Network Architecture version 3.0. In addition, several demonstration services such as multiparty high-quality audio and video conferencing, shared database access and subscription management services have been created.

To support the design and implementation of the MESH platform a DSC (Distributed Software Component) framework has been developed. This framework is a generalization and implementation of the TINA computational object model and can also be applied outside the TINA domain. The DSC framework acts as a middleware layer, which shields component designers from many communication level details. A DSC can be mapped to a computational object or object group. DSCs can be grouped to form compound components from sub-components that also can consist of multiple components, etc. In addition, the DSC framework addresses flexible configuration, dynamic component construction from (downloadable) sub-components, and dynamic interface instantiation.
\end{abstract}

The MESH platform not only demonstrates the potential of TINA, but also reveals several weak areas. This paper describes the DSC approach, which we used to design and implement major parts of TINA, and our experiences with TINA.

\section{INTRODUCTION}

During the MESH [1] (Multimedia services on the Electronic Super Highway) project, a TINA [2] (Telecommunications Information Networking Architecture) based platform for networked multimedia, multiparty services has been designed, implemented and evaluated.

In the MESH project a number of companies and knowledge institutes have developed pragmatic ways of working together on the electronic highway. The project focused particularly on multiparty, multimedia applications such as desktop video conferencing and co-authoring (electronic teamwork on the same document). The goal of MESH is to support teamwork in such a way that the natural, dynamic communication process between individuals remains intact.

The MESH project was sponsored financially by the Dutch Ministry of Economic Affairs
Several groups of people can work on a joint product at different locations and, if necessary, even at different times. They should feel as if they are all gathered in the same meeting room, with all the necessary facilities. During the MESH project, a number of services have been developed for the following application domains:

- Tele-consultation in the health care sector, for use by specialists at rehabilitation clinics,

- teamwork between lecturers at different universities,

- tele-learning for students at different universities,

- tele-meeting in a distributed organization.

MESH aims to bring the needs of future users and the opportunities of the marketplace together. To achieve this, all the important players are represented in this project. Suppliers of hardware and network services, such as Lucent Technologies, KPN Research and SURFnet. Users, such as the Academic Hospital of the University of Amsterdam and Roessingh Research and Development, Research institutes, such as the Telematica Instituut and the Centre for Telematics and Information Technology of the University of Twente.

An important objective of MESH was to design a platform architecture, which would be supported by open industry standards. This lead to the choice of TINA, which is an open software architecture supported by the world's leading network operators, telecommunications equipment and computer equipment manufacturers.

In this paper, we describe our approach and experiences with the design and implementation of the MESH services platform. In Section II we first give a description of a DSC [3] (Distributed Software Component) framework which has been developed within the MESH project to support the design and implementation of the MESH platform. This framework can be seen as the foundation on top of which the MESH implementation of the TINA service and network architecture has been build. In Section III we describe the MESH platform architecture and our implementation of various parts of the TINA service and network architecture. Finally, in Section IV, we draw some conclusions and give an overview of future work.

\section{THE DSC FRAMEWORK}

The Distributed Software Component framework has been developed within the MESH project with the goal to accelerate the design and implementation of the MESH platform. Frameworks have been describes as a technology for reifying 
proven software designs and implementations in order to improve the quality of software [4] The DSC framework is a concrete part of the platform and can be seen as the infrastructure that allows components to interact and collaborate. The DSC framework:

- Supports a Component Oriented Programming [5] (COP) paradigm,

- provides a runtime support environment,

- implements TINA's engineering viewpoint,

- supports TINA's computational viewpoint,

- provides development support tools with source code generation,

- provides runtime monitoring, tracing and debugging facilities.

$\mathrm{COP}$ is an increasingly popular modeling and implementation paradigm. COP has been described as a natural extension of object oriented programming to cater the needs of independently extensible systems $[5,6] \mathrm{COP}$ allows developers to concentrate on high-level application content, which improves the rate and productivity of development. Examples of this technology can be found in ActiveX components [7], OpenDoc [8], and JavaBean [9].

An important benefit of the component model is that it provides a higher level of abstraction compared to the object model and that it enables flexible software construction by combining and connecting individual components. The goal is to create a repository of multi-usable components that can be used for component-based software development. Software development then becomes the selection, adaptation, and composition of components.

The TINA architecture is also modeled as a set of collaborating components using the Reference Model for Open Distributed Processing (RM-ODP). RM-ODP was a joint effort by the ISO and ITU-T to develop a coordinating framework for the standardization of open distributed processing (ODP) [10]. RM-ODP aims to achieve portability between heterogeneous platforms, interworking between ODP systems, and distribution transparency, i.e. hide the consequences of distribution from both the application programmer and user. An excellent introduction to RM-ODP can be found in [11]. The DSC framework is a generalization and implementation of the TINA computational object model. Runtime support for the DSC framework provides a middleware layer, which shields component designers from many communication level details. A development environment supports the DSC framework and assist developers by generating component implementation templates from a formal component specification. The DSC framework also provides runtime testing, monitoring and debugging facilities. CORBA [12] (Common Object Request Broker Architecture) was used as the underlying Distributed Processing Environment (DPE) and the implementation was done using the Java programming language.
During the implementation of the MESH platform, the use of this framework has resulted in a significant productivity increase. The framework provides many lower level services, which allows developers to focus on high level tasks and responsibilities of the numerous TINA service components from which the MESH platform is build.

\section{A. Distributed Software Components}

To fit into the framework each component must support a set of common features. Components in the DSC framework are the basic building blocks from which complex systems can be constructed. Compound components can constructed by aggregated sub-components (see Section B ) with arbitrary levels of nesting. Non-compound components form a unit of distribution. They can operate on any physical node within the network provided it is accessible through the DPE. The distributed components can be located through a naming server or through references hold within other components.

Just as in the ODP model, components in the DSC framework have one or more operational interfaces, which allow access to the services the components offers. However, in the DSC framework all operational interfaces inherit also operations from a common i_operational interface. In addition, each DSC must also provide a single i component interface. This interface acts as the root access point to the component giving the component a unique identity and through which references to other interfaces can be obtained.

Each component must support a set of common services. These services are available through the i_component and the i_Operational interfaces. The i_component interface provides a standard interface for the following common services:

- Property services, with operations to read and define them,

- component life cycle services, with operations such as create, delete, suspend, and resume,

- transaction services, which allow the component to operate within the context of a transaction and with operations to commit or abort changes made,

- configuration services, which provide operations to support dynamic construction of compound components,

- debugging facilities, with which all invocations on an interface can be monitored.

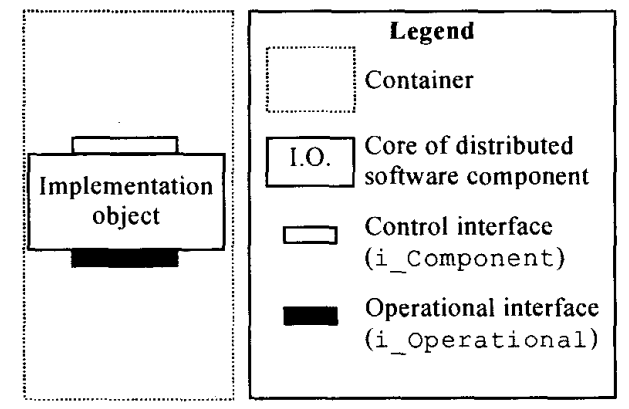

Fig. 1. Component symbology. 
In addition, components share common behavior, for example the ability to be notified of or to subscribe to events. Each component has a standard property named EventList, which contains the list of events that can be generated by the component. A client can register with the component as an observer for specified events. Clients also maintain the list of all the events for which they can act as an observer as a property.

The i_Component interface is the root access point to the component. A client component, which wishes to use the services of another component, must first obtain a reference to its i_Component interface. In the DSC framework, clients may obtain i_Component interface references through the CORBA naming service. Once the $i$ Component interface is obtained, operational interface references can be retrieved using the getoperational () operation.

The operational interfaces provide the service specific operations implemented by a component. In the TINA computational object model a component may have several operational interfaces where each operational interface is a group of closely related operations. This allows different clients to have a different perspective of a component. For example, a component might support interfaces supporting management services and interfaces supporting control services.

Fig. 1 shows a component as used in the DSC framework including the mandatory i_component interface and one operational interface.

\section{B. Compound Components}

A compound component is a (possibly nested) aggregate of sub-components, which, from an external view, is similar to single component, i.e. presenting the same set of operational interfaces, properties etc. One of the main strengths of the DSC framework is the ability to dynamically create such compound components. This allows the dynamic composition of complex components from simpler ones and stimulates the reuse of basic building blocks.

Compound components can be used to extend functionality of existing components. For example, a new operational interfaces can added to an existing component by creating a compound component which contains the original existing component plus a new component which implements the additional interfaces.

Compound components present a single i Component interface and a single identity to the external world. The i_Component interface is provided by the top level component. Client components obtain operational interface references for any of the sub-components through the i Component interface of the top level component. The top level component also defines which properties, interfaces, or events from sub-components are exported and visible at the compound level. Fig. 2 shows an example of compound component containing two components.

Compound components can be dynamically created or destroyed through operations provided by the i_Component interface of top level component.

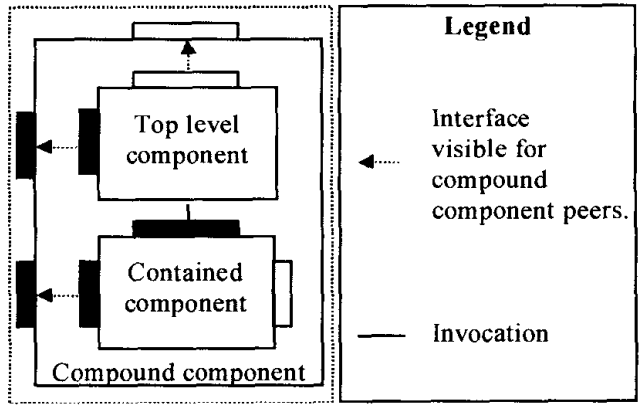

Fig. 2. Compound component.

These operations in the $i_{-}$Component interface are (in OMG IDL notation):

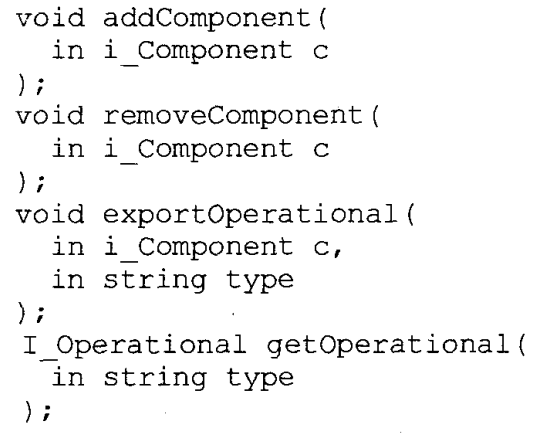

A compound component maintains a list of subcomponents. The operation addComponent will add the given sub-component reference " $c$ " to this list and the operation removecomponent will remove it from the list. The operation exportoperationaI will add the given interface "type", from the given sub-component "c" to a list of externally visible interface types, which is also maintained by the control component. Both these lists are available as properties and can be queried by other components.

In general, a property is an attribute of a component or an interface, which can be used to provide detailed information about the component or interface. A property has a name, type, and value. Within the DSC framework properties can have a component wide scope or a scope which is limited to an operational interface that contains the property. Component wide properties are access through the i_Component interface.

Properties can be used as configuration variables, for example to specify engineering attributes such as concurrency policies, interface names, event generation, component composition etc.

\section{Component Container}

Each component in the DSC framework belongs to a component container. The container is a specialized compound component, which provides the run time context in which components operate. In our implementation, the run time context includes the Java virtual machine and the Object Re- 
quest Broker (ORB). The container also controls concurrency policies, for example creating a thread pool of configurable size to allow concurrent access to the components within the container. The container itself is also a component with a $i$ Component interface and one operational interface $i$ Container. This interface is accessible by all components within the container. The container component allows new components dynamically to be added or removed. The i container interface provides an operation to create a new component instance of a given type within the container.

To be able to create a new component instance all necessary byte codes (in case of Java) must be available on the local machine. In our implementation component byte codes are packaged and distributed in Java jar archive files. To create a new component the container will first examine a local component repository for the availability of the requested component and instantiate the component from this repository if present. If the component is not present in the local repository, the container will contact the service provider and request all missing component packages to be downloaded into the local repository (see Fig. 3). After the download is completed the components can be instantiated. This process is completely transparent to the end user. The download process must take place within a secure context in which a trusted relationship exists between the end user and the service provider from which the components are downloaded.

\section{Component Specification Language}

The component specification language can be used to specify components. It can specify the initial topology of compound components and list per sub-component which of its interfaces are exported to the compound component. The specification includes properties and events that can be accepted or that fired from an interface or component. Interfaces can be specified to be dynamic, in which case a new object instance is created per getoperational () request, or static in which case a single object instance is associated with the interface. Interfaces operations are not specified in the component specification language; they are specified separately in OMG IDL.

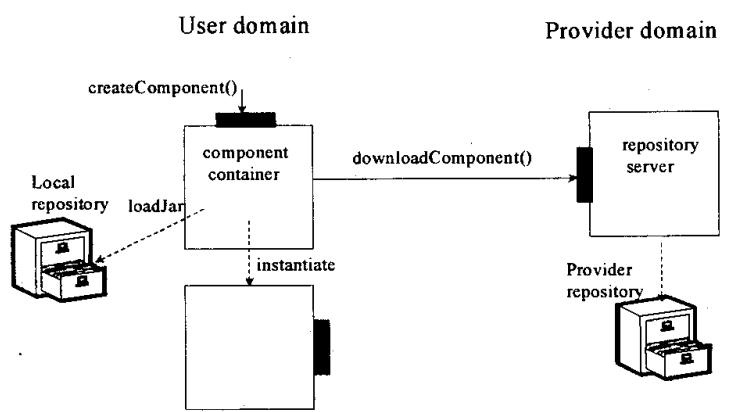

Fig. 3. Component downloading
The following fragment is an example of the component specification language. It specifies a compound component named myComponent, which contains a sub-component mySubComponent, which exports a single interface, an ExportedInterface. MyComponent further includes one operational interface, $i$ interfacel. This interface accepts an event named myEvent of type short. The component itself can also fire and accept one event.

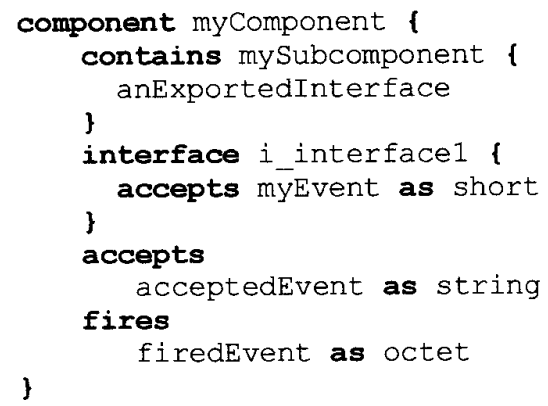

The component specification, combined with interface IDL specifications is used to generate source code implementation templates, which a developer must further complete. This process is explained in Section E.

\section{E. Component Development Environment}

Fig. 4 shows the component development process. During component development three separate stages can be identified: (1) specification of interfaces and events in OMG IDL, (2) specification of components in the component specification language, and (3) implementing the components behavior in any language for which there exists IDL bindings. We used Java [13] as an implementation language.

During the first stage, all required and supported (both static and dynamic) interfaces and all emitted and accepted event types are specified in OMG IDL.

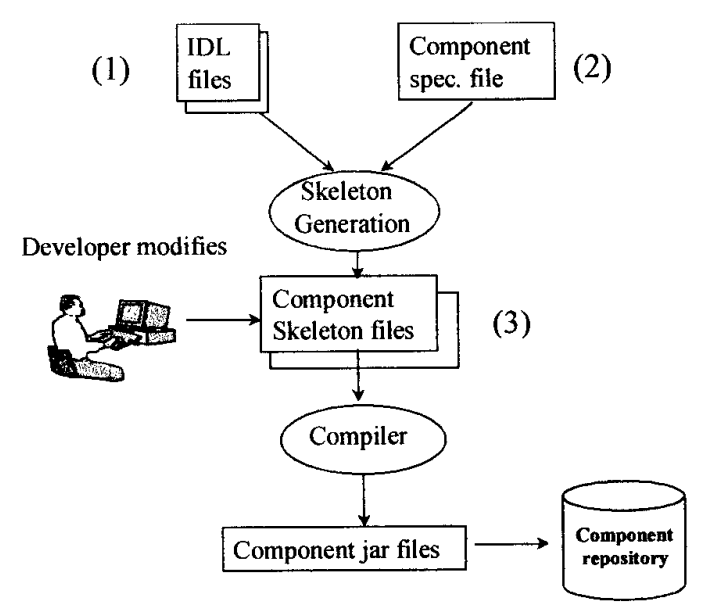

Fig. 4. Component development process. 
During the second stage, the component specification language is used. It relates interfaces and event types together to form a component. In addition, information about component composition, interfaces imported from sub-components, properties, incoming and outgoing event can be specified. A component skeleton generation tool is developed which processes OMG IDL files together with component specification files to generate a set of implementation skeleton files. The generated Java files contain code to start the static interfaces, to encapsulate components, export static interfaces, and to set the specified properties. Also, implementation skeletons, code needed to map events to JavaBean events, and intermediate debug source is generated per interface. All generated and modified files are compiled using a Java compiler and the resulting classes are collected in a JAR (Java Archive) file. A generated JAR file contains all required classes and resources needed at run-time.

The last stage consists of implementing and testing the behavior of the components. Except for the previously generated implementation skeletons, and debugging facilities, this stage is not further automated. The debugging facilities can be optionally activated per interface. They can be used to gradually monitor all invocations on an interface or to trace a sequence of invocations on subsequent interfaces. The later information can be graphically presented. It is especially useful to verify the dynamic behavior of the components with the message sequence diagrams found in the design documents.

\section{MESH SERVICES PLATFORM}

The DSC framework described in Section II has been used to implement the MESH platform. The MESH platform implements a large part of the TINA service architecture [14] version 5.0 and the TINA Network Architecture [15] version 3.0. In the current version of the MESH platform, the TINA roles of the Retailer and third party service provider have been combined into one service provider role. The service provider implements the full retailer reference point interface needed for both access and usage sessions, but not the retailer to retailer reference points. Future work may expand the implementation to include these reference points as well.

Fig. 5 gives on overview of the TINA service components that have been implemented in the MESH platform. The components within Fig. 5 are grouped in several domains. The consumer domain contains all components that ca be instantiated at the end-user terminal. The service provider domain contains all components that are instantiated at one or multiple service provider nodes within the network. The connectivity provider domain contains the components that are used to set-up streambindings between end-users.

The architecture also consists of four distinct levels. The access session level contains all the components that play a role during an access session. The access session level is described in subsection A subsection B describes the service level components, subsection $\mathrm{C}$ describes the communication level components and finally, in subsection D the connectivity level components are described

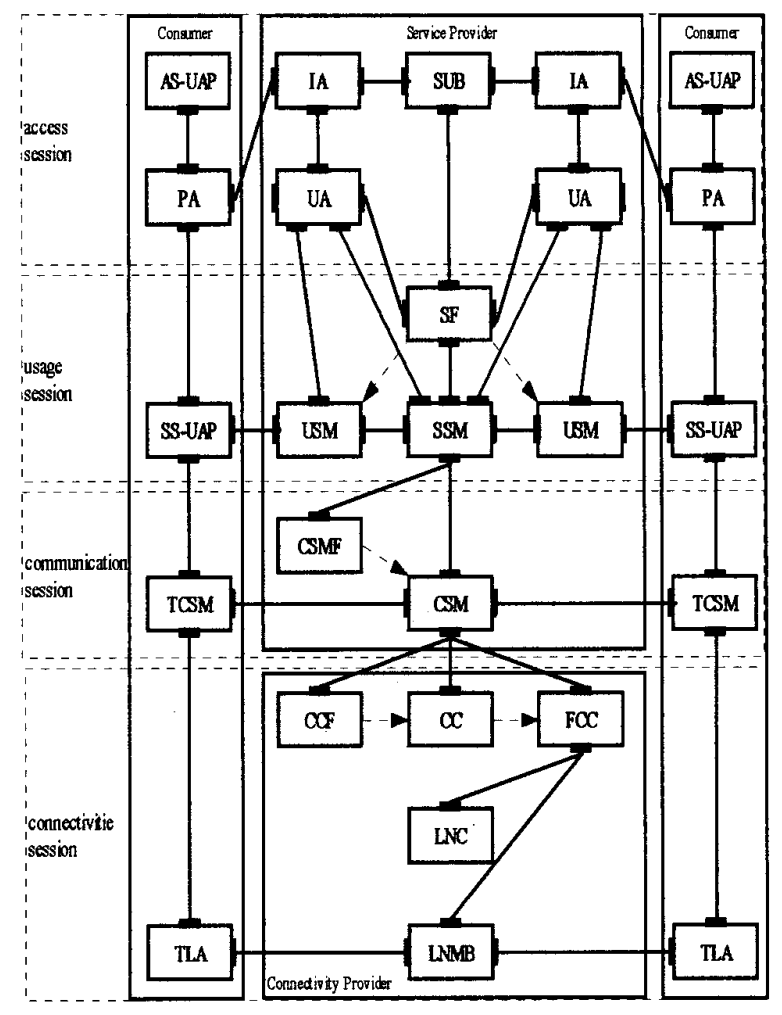

Fig. 5. MESH architecture overview

\section{A. Access Level}

In the TINA architecture, all interactions between a user and a provider are executed within the context of a session. The architecture distinguishes between an access session and a service session. The access session is used for the identification of the user and the establishment of the terminal used during a service session. After the access session is successfuily completed, the user can start a service session in which he can select one or multiple services to use.

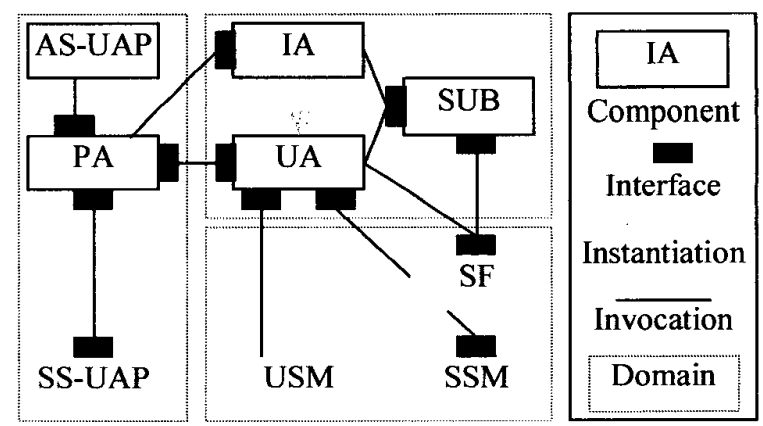

Fig. 6. Access level architecture components. 
Fig. 6 shows the components that play a role during the access session. These are the Access Session User Application (AS-UAP), the Initial Agent (IA), the Provider Agent (PA), Subscription Management Component (SUB) and the User Agent (UA). The AS-UAP contains a graphical user interface, which will prompt the user for identification and authentication. The PA is used by the AS-UAP to communicate with the provider through the IA. The IA authenticates the user using the SUB to obtain subscription information and starts a UA. Together the PA and UA establish a secure and personalized access session that allows the user to select and start any service for which he or she has a subscription.

Before a user can start an access session he or she must first have all necessary software installed on his or her local machine. In the MESH project, this bootstrap process is solved using an installation procedure that can be started through a common web browser. In this scenario, the service provider runs a web server with a home page through which an installation process can be started. When the end-user accesses this home page, he may choose to start the MESH installation. The home page contains a Java applet, which downloads all necessary software to an installation directory of the users choice (and for which he must have granted security permissions).

Once the software has been downloaded, an access session will be started. The user can now login as an anonymous user and start a subscription service. The subscription service will allow the user to fill in personal account data such as a login name and password. The subscription service also allows the user to subscribe to a set of services that the service provider offers. The subscription service can also be used later to change the selections.

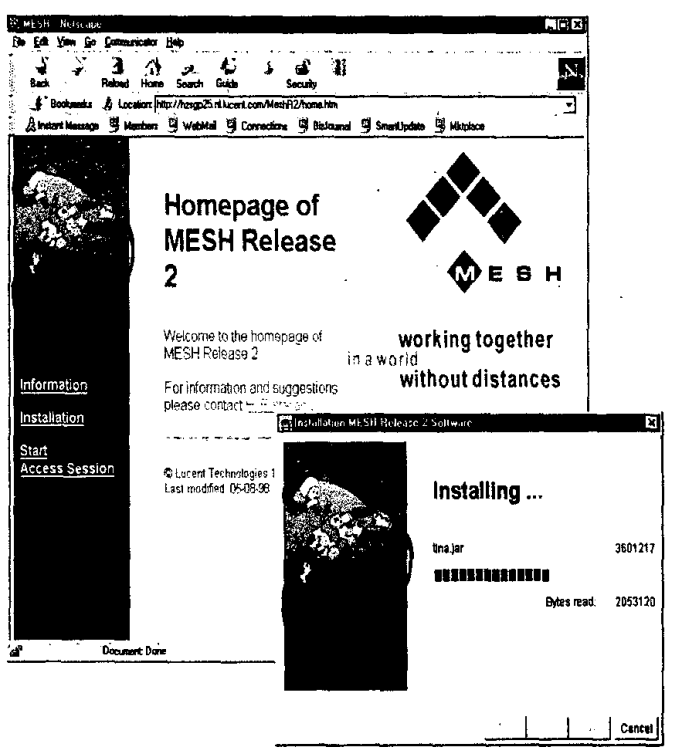

Fig. 7. Browser activated installation

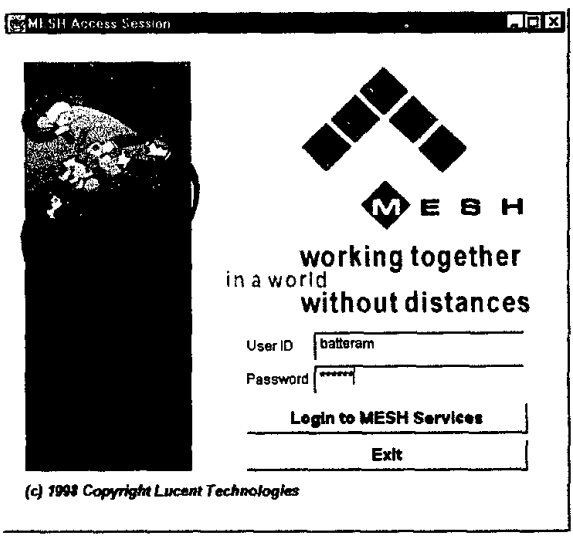

Fig. 8. Access session login dialog.

After the user has created an account, he or she can start to use the services for which he or she has a subscribtion. First a new access session must be started using the new account. Once the initial software has been downloaded to the user's terminal, subsequent access sessions can be started as standalone applications or within a browser context, whichever the user prefers.

When the user starts a new access session, the user may see a list of previously suspended sessions, which the user may choose to resume. In addition, a list of active sessions to which the user has been invited can be shown. The user can accept or decline each of the invitations. If an invitation is accepted the service specific user application (SS-UAP) for that service will be started and the user is joined to the session. During an active session, new invitations may arrive which the PA handles by popping up a dialog window giving the user the choice to accept or decline the invitation as shown in Fig. 9.

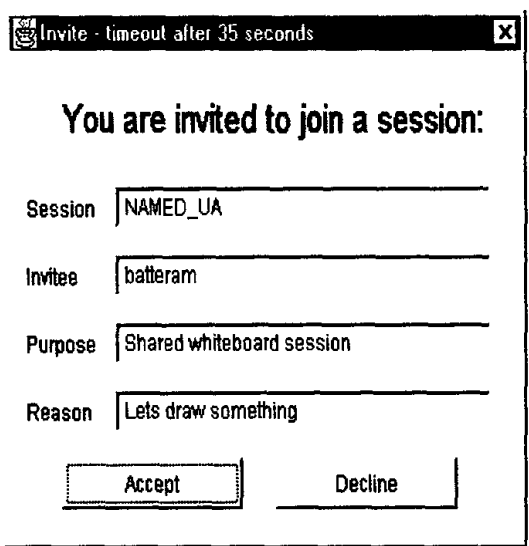

Fig. 9. Invitation dialog. 


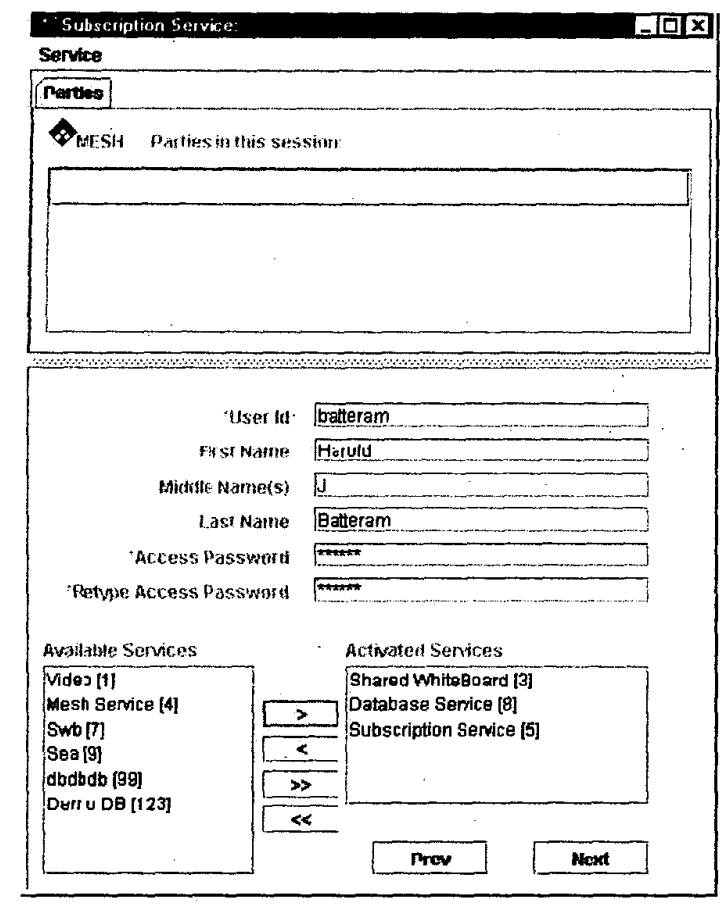

Fig. 10. Subscription service.

\section{Subscription Management}

The Subscription Management Component (SUB) in MESH provides functionality to manage the subscription information model for the whole set of services in the Service Provider domain as defined in [14]. It is implemented with compliance to the suggested TINA model for a subscription management component.

The SUB interacts with other components mainly during the access session (see Fig. 6). The IA contacts the SUB to retrieve user subscription information during the user authentication process. The UA also interacts with the SUB component to retrieve user information, obtaining or storing user properties, etc. Since the SUB also contains the description of the services, the Service Factory (SF) contacts the SUB during the usage session and retrieves all the information needed for the proper instantiation of service specific components.

The SUB component is a compound component, consisting of two loosely coupled-sub-components, a SUB and a Database Management component (see Fig. 11). This separation serves two purposes: (1) to ensure independence from a particular DBMS and (2) to allow distribution of the workload; e.g., the Database Management component can be run on a dedicated machine. Since the Database Management component interacts only with the SUB it is treated as an encapsulated part of the compound SUB.

The SUB allows the management of subscribers, service contracts between subscribers and services and entities such as users, terminal and network assignment points (NAP) for the complete set of services provided by a provider. The main features provided by this component are:

- Creation, modification, deletion and query of subscribers,

- creation, modification, deletion and query of subscriber related information (associated end users, end user groups, etc.),

- creation, modification, deletion and query of service contracts (definition of subscribed service profiles),

- retrieval of the list of services, either the ones available in the provider domain or the subscribed ones,

- retrieval of the service profile (SAGServiceProfile) for a specific user (or terminal or NAP).

All interfaces of the SUB component as proposed by TINA have been implemented. Several interfaces required modifications to support missing, essential functionality. Several inconsistencies, which were discovered during the implementation, are summarized in the next section. The internal architecture has been implemented as suggested by the TINA documentation with only minor changes.

The Subscription Coordinator (SCoo) sub-component is responsible for the management of the other sub-components as well as being a main control point for the functionality of the whole SUB. It coordinates the subscriber management and the service contract management. The SCoo also implements interfaces that are exported/visible outside the SUB and through which clients of the SUB can initiate interaction with the SUB, create new subscriber, contract services to a subscriber, list services, etc. The SCoo uses the Subscriber Management (SubM) sub-component for managing the subscribers and the Service Contract Managers (SCM) subcomponents to manage the service contracts.

The Subscriber Management sub-component (SubM) is responsible for the management of a pool of Subscriber Objects (SubO) - one per subscriber - that implement interfaces for managing entities (users, terminals, nap) and subscription assignment groups within a subscriber.

There is one Service Contract Management (SCM) subcomponent per service in the provider domain. An SCM is responsible for managing a pool of Service Contract Objects (SCO), one per subscriber, contracted the particular service.

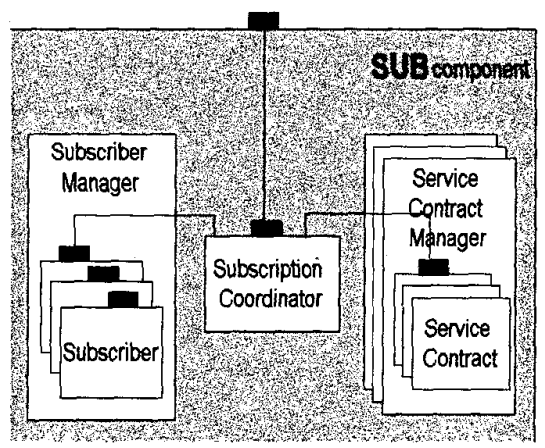

Fig. 11. Internal structure of the SUB component. 
Each SCO implements interfaces for manipulating service contracts and service profiles.

In our experience we found the model as suggested by TINA to be quite usable. It is a flexible model that allows easy and straightforward approach with the management of user oriented subscription information model. The suggested software component decomposition allows a dynamic implementation that, once instantiated, can be easily controlled.

During the implementation of the SUB component, several problems and inconsistencies in the TINA documentation were encountered. The following list summarizes the most important ones:

- The description of the service profiles scheme is not consistent. Special subscription assignment groups can be created to group entities (users and terminals) and to assign service profiles to groups. The documentation also describes that the entities could be assigned service profiles directly. However, on p.244 of Annex 3, it is explained that there is a third way, a user profile assigned to a user which does the same as the service profiles. Here we had to make a decision in order to be able to implement a good service profile model.

- Incomplete interfaces: We had to make changes to the original TINA interface definitions - The I SubscriberLCMgmt and I_ServiceContractLCMgmt interfaces were only described but not prescribed. We had to add in a number of operations that we needed for the communication between the internal sub components within the Subscription Component. The I SubscriberInfoMgnt interface was extended with several operations since the original TINA IDL specification was not expressive enough. An additional I serviceMgmt interface was defined to provide additional service manipulation features to the SUB. Originally, this role had to be done from the Service Life Cycle Management component (SLCM). However, since this component was not implemented, the SUB was extended to meet the requirements.

- Some structures (for example $t$ SAE) in the described information model only operations for creation/deletion but no accesses operations were provided.

- Some operations definitions lead to poor performance. For example, operations which copy big structures from the remote objects. It is more preferable to decompose these operations into several which fetch small parts of the structures since the most frequent need is not the whole structure.

\section{B. Service Level}

At the service level of the architecture, single or multiparty service sessions can be started and stopped and stream bindings for continuous data streams can be setup to communicate with each other.

Fig. 12 depicts the service level TINA components in a two-party service session. At the service provider, the Service
Factory (SF) creates, upon request by the UA, the Service Session Manager (SSM) and the User Session Manager (USM). The MESH platform supports only a single SF that creates service components for each service provided via the platform. The SF contacts the SUB component to obtain the names of the service specific SSM and USM components to be created for a given Serviceld. The reason for using a single SF is simply because there was no need for multiple SFs in our implementation. When the number of services used on the MESH platform becomes difficult to handle by a single SF, extra SFs can easily be added.

In the end-user domain, the Service Session User APplication (SS-UAP) is created by the PA. The SS-UAP present the service to the end-user.

The SSM maintains the global view of the session and contains the entire session model of parties, stream bindings and resources in the session. Thus, the session model is not distributed over the SSM and all the USMs. The reason behind this design decision is that consistency of the session model is much easier to maintain and that the SSM is the single point of control of and access to the session model information.

The MESH platform only supports the TINA session model. Thus, a session consists of parties, streambindings, control session relationships and so on. Consequently, the session model is fixed and there is no negotiation about session model support by a service during the start service scenario. The USM only serves as a security guard for controlled access to the SSM and as a service hatch to the proper SSUAP.

The service level components within the MESH platform support all the feature sets described in the TINA Ret Reference Point Specifications 0.7 as far as IDL specifications of the interfaces were made available by TINA-C. These interfaces are:

- BasicFS: to support end and suspend session requests. Allows the party domain to discover interfaces supported by the session.

- BasicExtFS: to allow the provider domain to discover interfaces supported by the party domain components.

- MultipartyFS: to allow the session to support multiparty services, such as information on other parties, ending/suspending a party in the session, and inviting a user to join the session.

- MultipartyIndFS: to allow the session to indicate requests that are to be processed to the party components.

- VotingFS: to allow parties to vote in order to determine if a request should be accepted and executed.

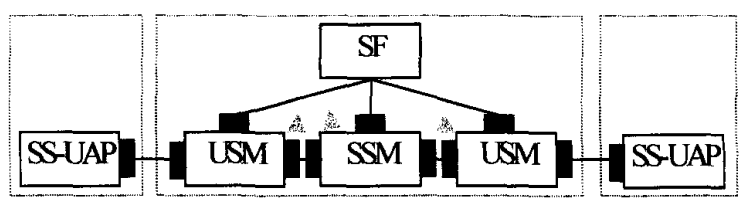

Fig. 12. Service level architecture components. 
- ControlSRFS: to support parties having ownership and $\mathrm{read} / \mathrm{write}$ rights on session entities (i.e. parties, resources, stream bindings, etc.).

- ParticipantSBFS: to provide high level support for setting up stream bindings in terms of session members participation.

- ParticipantSBIndFS: to provide participant type stream bindings with indications.

Announcement of service sessions is not yet supported by the MESH platform and thus all parties have to be explicitly invited to a service session. Adding and removing of resources to a service session is done using a non-TINA interface at the SSM, because at the time of writing the ResourceFS was not yet standardized by the TINA-C.

Within the MESH platform a stream binding consists of a number of uni-directional Stream Flow Connections (SFCs) to which some or all of the participants are bound. A SFC consists of a set of Stream Flow End Points (SFEPs), one per participant in the SFC. All SFEPs in a SFC have the same binding tag. Consequently, the binding algorithm executed by the SSM can be kept relatively simple. It only has to match SFEPs with similar binding tags. When the SSM has bound all the SFEPs to SFCs, it interacts with components at the communication level to actually setup the SFCs.

In our development approach, specific services are build on top of the service level components of the TINA architecture, in particular the SS-UAP, SSM and USM. These components provide generic service session management functionality that is necessary in each service. In particular, with this generic service session management functionality, service sessions can be started and deleted, and participants and stream bindings can be added to a service session, modified and deleted from it. In our approach, any service is build by extending the generic SS-UAP, SSM and USM components with service-specific functionality. A service then consists of service-specific versions compound components of the SSUAP, SSM and USM components that encapsulate the generic SS-UAP, SSM and USM as sub-components. In Fig. 13, an example service-specific SSM is depicted.

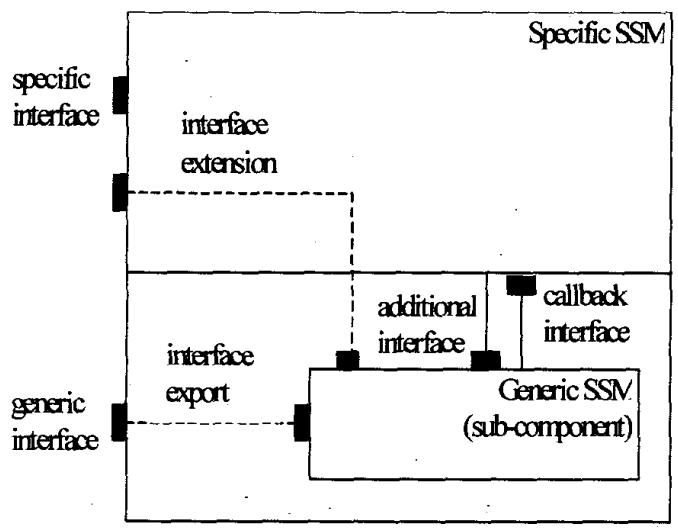

Fig. 13. A service-specific SSM.
The DSC framework enables a component to export an interface of one of its sub-components. Thus, the servicespecific SS-UAP, SSM and USM can export some or all of the interfaces of the generic SS-UAP, SSM and USM depending on which interfaces or feature sets are required by the service. On the other hand, the service-specific components can overload or extend operations of their generic subcomponents in order to perform service-specific actions. For example, for a database service, the initialize operation of the i_Init interface of the specific SSM might extend the generic initialize operation to open a database that is used during the service. Obviously, the service developer has to use this feature with care in order not to disable generic functionality that is vital for proper service behavior.

Besides exporting/extending interfaces of the generic subcomponents, the service-specific components can additionally provide for interfaces with operations that implement servicespecific functionality. To allow these operations to fully use the functionality of the generic sub-components, extra internal interfaces at the generic SS-UAP, SSM and USM have been defined. In addition to the TINA specified interfaces we had to define two new interfaces:

- Interface $i$ SessionModel that allows the specific SSM to query the session model that is maintained in the generic SSM. This interface also allows the specific SSM to modify the session model in case that is not possible via the TINA interfaces. For example, the i sessionModel interface of the SSM allows for the addition and removal of resources to the service session, because this is not part of the TINA Service Architecture 5.0.

- Interface i GenericSSM that allows the specific SSM to apply for globally unique identifiers, to obtain references to interfaces of other components, and to register a callback interface i_SpecificSSM of the specific SSM.

The generic USM has one extra interface:

- Interface i_GenericUSM that allows the specific USM to obtain references to interfaces of other components, to check the secretID provided by the specific SS-UAP and to register a callback interface i_SpecificUSM of the specific USM.

The SS-UAP has two extra interfaces:

- Interface $i$ SessionModel that allows the specific SSUAP to get session model information that is maintained in the generic SS-UAP.

- Interface $i$ GenericsSS-UAP that allows the specific SS-UAP to obtain references to interfaces of other components, and to register a callback interface i_SpecificSS-UAP of the specific SS-UAP.

The callback interfaces of the specific components provide operations that can be called by the generic sub-components upon initialize, suspend, resume and end of a service session. Although registering a callback interface is not obligatory, there is one requirement that each specific service has to satisfy: the i_SpecificSS-UAP interface must be registered 
with the generic SS-UAP and this interface must implement a startservice operation in which the service-specific SSUAP is started.

Besides callback interfaces, a specific component and its generic sub-component can interact via an event-listener mechanism. In particular, the specific component can register itself for certain events within the generic sub-component. Especially within the generic SS-UAP, various events occur in which the specific SS-UAP might be interested. These events occur as a result of indication and information messages from the USM/SSM. They include invitations, the addition, modification and deletion of participants, stream bindings, and indications on which a vote is required.

\section{Communication Level}

The components within the communication level manage the communication network and control the communication sessions. A communication session provides a serviceoriented view on the stream bindings between the participants of the session. Typically, the service session specifies a stream binding to be set up between parties in QoS parameters and abstract medium descriptions. The communication session encapsulates the details involved with the process of matching the terminal specific communication characteristics (such as codecs, audio and video capabilities, etc.) upon the requested QoS (quality of service).

There are three components that play a role in a communication session: the Terminal Communication Session Manager (TCSM), the Communication Session Manager Factory (CSMF), and the Communication Session Manager (CSM), see Fig. 14. The TCSM is part of a user's terminal; it manages the communication characteristics of the terminal and controls the bindings within the customer premises. The CSMF is a factory for CSMs. The CSM controls the network part of the communication session, where the TCSM controls the terminal part of the communication session for each party only.

The CSM controls the individual elements of the network communication session. The terminal-specific part of the communication session is controlled by the involved TCSMs. The CSM is responsible for combining the stream flow end

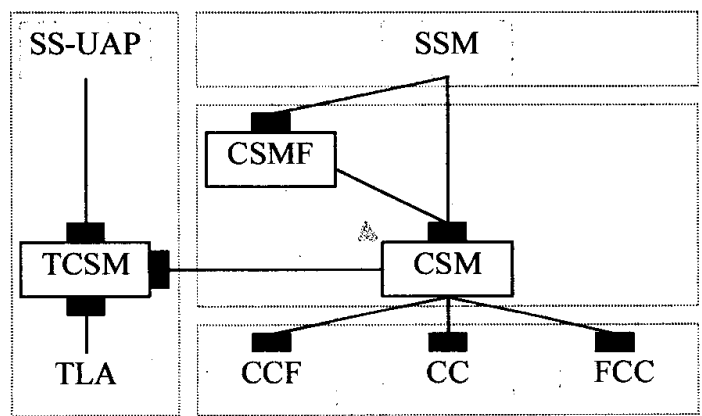

Fig. 14. Components involved in the communication session. point capabilities into a stream flow connection, all capabilities have to match, or a special resource that can translate the capabilities needs to be available. Inspecting the QoS parameters and the capabilities results in constraints for mapping logical stream flows into physical network flows.

The TCSM manages the communication characteristics of the terminal. It maps general medium descriptions with QoS parameters into stream flow end points that match the request of the SS-UAP. Each stream flow end point has additional communication capabilities. Besides codec configuration, a communication capability might state connectivity requirements. For example it might state that this stream flow end point is based upon RTP (Real-time Transport Protocol) and it is best used upon a UDP (User Datagram Protocol) binding which is, in turn, best used upon an IP (Internet Protocol) layer network. These requirements are input for the CSM to choose a connectivity provider with whom the service provider has a contract profile that allows the control of the specified physical network flows.

A full specification of the interface between the SS-UAP and the TCSM was not available; this interface is part of the terminal intra-domain reference point. Through this interface the SS-UAP queries the TCSM for available stream flow end point descriptions based upon a high-level medium description. A simple interface supporting our target scenarios has been specified in this project. Also, the TSCM supports the i Terminalcomssetup and the i_TerminalComSCtrl interfaces. The former interface supports querying for the capabilities of the stream flow end point descriptions. Yet, in specified operation it is not clear which capability is related to which stream flow end point description. Consequently, we slightly modified the operations to reflect the relation between capabilities and stream flow end point descriptions.

\section{Connectivity Level}

The components within the connectivity level manage the connectivity network and control the connectivity sessions. The connectivity session hides the network technology related details towards the communication session and the service session. An example of a connectivity detail is whether a network supports uni-directional of bi-directional bindings. The communication session models stream flow connections always as unidirectional, but the connectivity session can support multiple stream flow connections using only one network flow connection depending on the network capabilities.

One connectivity session might span multiple connectivity networks, provided special resources that map connectivity details between different connectivity networks are available [16]. Neither the service session nor the communication session is aware of this.

Fig. 15 shows four components part of the connectivity level: the CCF (Connection Coordinator Factory), CC (Connection Coordinator), the FCC (Flow Connection Controller), and the Layer Network Controller (LNC). The CCF is a factory for CCs. The $\mathrm{CC}$ sets up and controls the entire connec- 
tivity session. A connectivity session consists of network flow end points and network flow connections. Each network flow connection is set up and controlled by a separate FCC. The CC instantiates a FCC per request for a network flow connection. The FCC contacts LNCs to claim and use resources in their layer networks that make up the actual bindings. A layer network can contain multiple administrative domains and is typed by the supported types of bindings. An LNC sets up and controls bindings through one administrative domain of a layer network.

Before compiling the prescriptive connectivity level interfaces, we had to change the NFEP definitions. NFEPs are maintained by TLAs. A TLA is layer network specific. Layer network type specific components contact the corresponding TLA that offers the NFEPs. Therefore, the TLA its interface reference has to be available. Originally, there were two NFEP definitions i.e., $t_{-}$ANfep and $t_{-} N f$ epDesc, where $t_{-} N$ fepDesc contains a $t_{-}$ANfep and where $t_{-}$ANfep supports recursive NFEP specification. Neither of them contained a TLA interface reference. We combined both definitions into a new $t$ NfepDesc and we created a separate $t \_N f e p P o o l D e s c$. The former contains a TLA interface reference field and the latter contains a sequence of $t_{-}$NfepDescs. Therefore, our NFEP definition did not support recursion. We had to drop the recursion requirements since the used IDL compiler did not support this.

Additionally, the prescriptive connectivity level interfaces contained a security parameter per method, a $t$ secHandle. This t_secHandle was defined as a long. Clearly, a long is neither a flexible security parameter nor a future proof solution. It is even questionable whether it is good practice to enforce a security procedure that demands per call authentication. Rather than solving such issues on connectivity service level, it should be solved by procedures running in parallel with a connectivity session. Establishing and maintaining a secure context between stakeholders is the responsibility of the connectivity access session that is executed before requesting a connectivity service.

To get the proposed descriptive components running we had to add interfaces to CCF, CC, and FCC. Unlike the CSMF, that controls the life cycle of the CSM, the CCF and $\mathrm{CC}$ are not explicitly involved if one of their spawned components is released; the administration of the CCF and CC

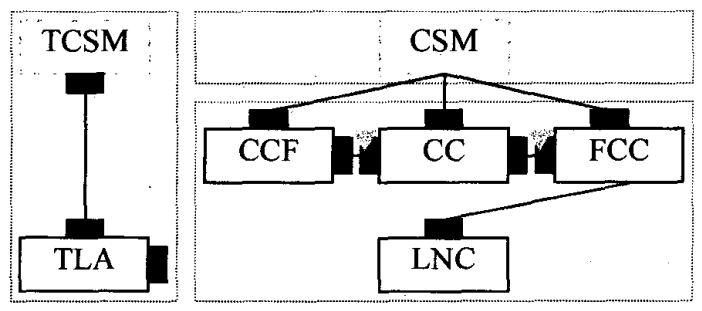

Fig. 15. Network resource architecture components. The layer network-type specific components were omitted. might not be consistent. We have extended the CCF and $\mathrm{CC}$ with a notification interface that accepts messages of a child-component that is about to be released. In addition, the $\mathrm{CC}$ and FCC components are extended with component constructor interfaces that enable their parent components to construct and initialize them.

\section{CONCLUSIONS AND FUTURE WORK}

In our experience, the TINA architecture is complex, extensive, and still immature. Several TINA reference points are incomplete and others are not yet specified, such as the LNFed and CSLN reference points. However, during the design and implementation efforts it proved to be conceptually sound. In our opinion, the TINA access and service levels are the maturest.

During January 1998 we obtained the prescriptive IDL specifications for the descriptive components from [17] and [18]. We noticed three IDL coding styles: one for the service and access level interfaces, one for the communication level interfaces, and one for the network level interfaces. Each style differed in module policy, distribution of interfaces over modules, and in include file approach. Consequently the interfaces where hard to read. Not only there where cosmetic problems to overcome, also the IDL code per style featured different naming conventions. Both integers (unsigned long), single strings, and sequences of strings ( $t$ _T inaName) where mixed. In order to stick to one coding style and naming convention; we modified the IDL files appropriately.

A general coding experience is that much time was lost coding the processing of the complex arguments of the specified operations. We recommend generating object-oriented code for the processing of complex arguments like $t$ Capabilityset or $t$ TinaName.

The DSC framework and support tools have played a significant role in the implementation of the MESH platform. It has accelerated the implementation process through template generation and by providing a comprehensive runtime environment which offers many common services such as software downloading, dynamic component composition, component configuration, and distribution transparencies. It also accelerated the testing and debugging process through automated generation of test components and runtime diagnostic services such as interface analysis and call flow analysis.

Future work will expand the implementation of the MESH platform in the following areas:

- Large scale deployment with scalability, load balancing and fault tolerance,

- accounting and billing services,

- service creation through component composition and specialization with graphical software tool support,

- new services for electronic commerce, medical and educational sectors.

These activities will be done in a new project named FRIENDS (FRamework for Integrated Engineering and Deployment of Services), starting January 1999. The project 
partners are Lucent Technologies, the research arms the Dutch telecom operator KPN, the Dutch Telematic Institute, the Dutch National Organization for Applied Scientific Research (TNO) and the University of Twente (CTIT).

\section{ACKNOWLEDGMENT}

We thank all contributors who made the MESH project a success. The views expressed in this paper are those of the authors, and not necessarily those of the other MESH project partners.

\section{REFERENCES}

[1] MESH, see http $/ /$ www.mesh.nl/

[2] TINA-C, see http $/ /$ www.tinac.com/

[3] Bakker, J.L., and H.J. Batteram, "Design and evaluation of the Distributed Software Component Framework for Distributed Communication Architectures", Proceedings of the $2^{\text {nd }}$ international Workshop on Enterprise Distributed Object Computing (EDOC'98), 98EX244. IEEE, pp. 282-288, San Diego (USA), November 3-5, 1998. (ISBN: 0-7803-5158-4)

[4] Mohamed E. Fayad and Douglas C. Schmidt, "Object Oriented Application Frameworks", Communications of the ACM, October 1997 , volume 40 , number 10 , pp 32-38.

[5] International Workshop on Component-Oriented Programming; Jyväskylä Finland, 1997, see:

http:/www.ide.hkr.se/ -bosch/WCOP97/papers.html
[6] International Workshop on Component-Oriented Programming; University of Linz, Linz Austria; 1996, see: http $/ /$ www.ide.hkr.se/ bosch/WCOP97/WCOP.96.report.ps

[7] ActiveX, see http:/www.microsoft.com/

[8] Orfali, R., D. Harkey, and J. Edwards, The essential distributed objects survival guide, Wiley, New York (NY) USA, 1996.

[9] JAVA Beans, see http//java.sun.com/

[10] International Standards Organisation, Basic reference model of Open Distributed Processing - Part 1: Overview and guide to use, Standard ISO.IEC 10746-1, 1995.

[11] Raymond, K., "Reference Model of Open Distributed Processing; introduction", Proceedings of the $3^{\text {rd }}$ IFIP TC6/WG6.1 International Conference on Open Distributed Processing, pp 3-14, Brisbane (Australia), February 20-24, 1995.

[12] OMG/CORBA, see http:/www.omg.org/

[13] Java, See: http:/java.sunsoft.com/

[14] TINA-C, Service Architecture, Kristiansen, L. (ed.), TINA-C, Redbank, NJ (USA), June 1997

[15] TINA-C, Network Resource Architecture, Steegmans, F. (ed.), TINA-C, Redbank, NJ (USA), February 1997.

[16] Bakker, J.L., and F.J. Pattenier, "The Layer Network Federation Reference Point, Definition and implementation", The Application of Distributed Computing Technologies to Telecommunications Solutions (TINA'99), Kahuku-Oahu, Hawaii (USA), April 17-20, 1999. In press.

[17] TINA-C, Network Components Specification, http://tinac.com/1/97/resources/network/docs/ncs/v2.2/idl/modules.

[18] TINA-C, Service Component Specification: Computational Model and Dynamic, http://tinac.com/1/97/services/docs/scs/compmod/final/idl/. 